\title{
Incorporating airway examination photography into the electronic record
}

Joshua W. Sappenfield, Nik Gravenstein, Judith M. Wishin, Chukwudi Obiora Chiaghana, David Smyth, Brenda G. Fahy, Terrie Vasilopoulos, Laurie Davies, F. Kayser Enneking

Department of Anesthesiology, University of Florida College of Medicine, Gainesville, FL, USA

\begin{abstract}
Background. Photography of the airway has been used in research to validate preoperative airway assessment and the likelihood of identifying the difficult-to-mask ventilate and/or intubate patient. Up till now, no study has demonstrated the perceived utility of incorporation of airway photographs into the anesthesia preassessment.

Methods. The University of Florida Health Presurgical Clinic routinely incorporates three photographs of all adult patients during their preanesthesia visit. The first is a head-on view of the patient opening the mouth widely as part of a Mallampati examination, and the second and third are side views of the patient prognathing and with the neck in maximal extension, respectively. After IRB approval, providers of anesthesia were surveyed regarding their opinions on the perceived value of the new process. Chi-square tests were used to determine if the responses to each question significantly differed from the distribution that would be predicted by chance. $P<0.05$ was considered statistically significant.

Results. The survey was emailed to 180 individuals, with 145 responding. The responses significantly $(P<0.0001)$ indicated that the photographs helped the providers plan care for their patients and improved their satisfaction with the preoperative assessment. Technical and educational barriers were overcome using iterative Plan-Do-Study-Act cycles and coaching, respectively.

Conclusions. Photographs of the airway assessment can successfully be taken and incorporated into an electronic medical record in a busy presurgical clinic. The pictures provide additional perceived value to the traditional written assessment of a patient's airway examination by someone else.
\end{abstract}

Keywords: airway, electronic medical record, airway photography

\section{Introduction}

If "a picture is worth a thousand words", why then is the modern airway assessment still verbal and not visual? Three decades ago, Mallampati and others [1] described a link between visualization, or lack thereof, of oropharyngeal structures and the relationship of those findings to relative ease or difficulty with sub-

Address for correspondence: Joshua W. Sappenfield Dpt of Anaesthesiology Univ. of Florida College of Medicine 1600 SW Archer Road, PO Box 100254 Gainesville, Florida 32610 USA E-mail: jsappenfield@anest.ufl.edu sequent laryngoscopy. In 1987, Samsoon and Young [2] modified the "Mallampati" score and validated the scoring method using photographs of each participant's airway. Since then, the "Mallampati" score has remained the most widely used and studied airway examination to assess a patient's relative risk of being difficult to intubate. More recently, Connor and Segal [3] described computerized analysis of patient photographs to help assess the risk of a difficult intubation. They concluded that "anesthesiologists derive useful information from patients' facial appearance that improves diagnostic accuracy in predicting difficult intubation compared with the best available bedside airway tests alone" [3]. To evaluate whether or not implementing digital photography and integration into the electronic medical record was feasible, this initiative 
was implemented in UF Health's Presurgical Clinic and the end user was queried on whether they felt it added value. By integrating digital pictures into the record, anesthesia providers could make their own evaluation instead of relying on another individual's assessment. The hypothesis was that integration of digital pictures into the electronic anesthesia preoperative evaluation for patients would not only be possible but would improve the perceived value of a preanesthetic assessment done by someone other than the person scheduled to care for the patient in the operating room.

\section{Methods}

Obtaining photographs of the patients' airway examination and entering them into the patients' electronic medical record (EMR) have become a routine practice at UF Health's presurgical clinic since 2015. Patients arrive at the presurgical clinic and are registered by a medical technician (MT). The MT assesses the patients' vitalsigns and obtains a set of airway assessment photographs (Fig. 1) using an iPad mini 2 (Apple Inc., Cupertino, CA). The photograph in Figure $1 \mathrm{~A}$ is taken with the patient sitting in a chair and the iPad level with the patient's mouth. The patient is asked to open his/her mouth maximally with the tongue out. The picture is taken within 6 inches of the patient's mouth. The photographs in Figures 1B and $1 \mathrm{C}$ are taken from the patient's side to demonstrate his/her ability to prognath (the patient is requested to bite his/her upper lip) and maximally extend the neck without pain.

EPIC Canto (Epic Systems Corporation, Verona, WI) software is installed on the iPads, which allows the MTs to access the patient's EMR and directly upload images there. No patient information or photographs are stored on the iPad devices, thus ensuring the process is Health Insurance Portability and Accountability Act (HIPAA) compliant. The provider performing the preoperative assessment attaches the airway assessment photographs to the physical examination portion of the preoperative anesthesia assessment in the EMR. The anesthesia team scheduled to provide intraoperative care can then access the images in the EMR any time it opens the electronic chart.

Approximately 10 months later, we implemented our IRB-approved (\#2016-U-0021) request to email a survey to obtain anesthesia providers' (anesthesiology residents, certified registered nurse anesthetists, anesthesiology assistants, and attending anesthesiologists) feedback on incorporation of the airway assessment photographs into the anesthesia preoperative assessment they access via the EMR. Written consent

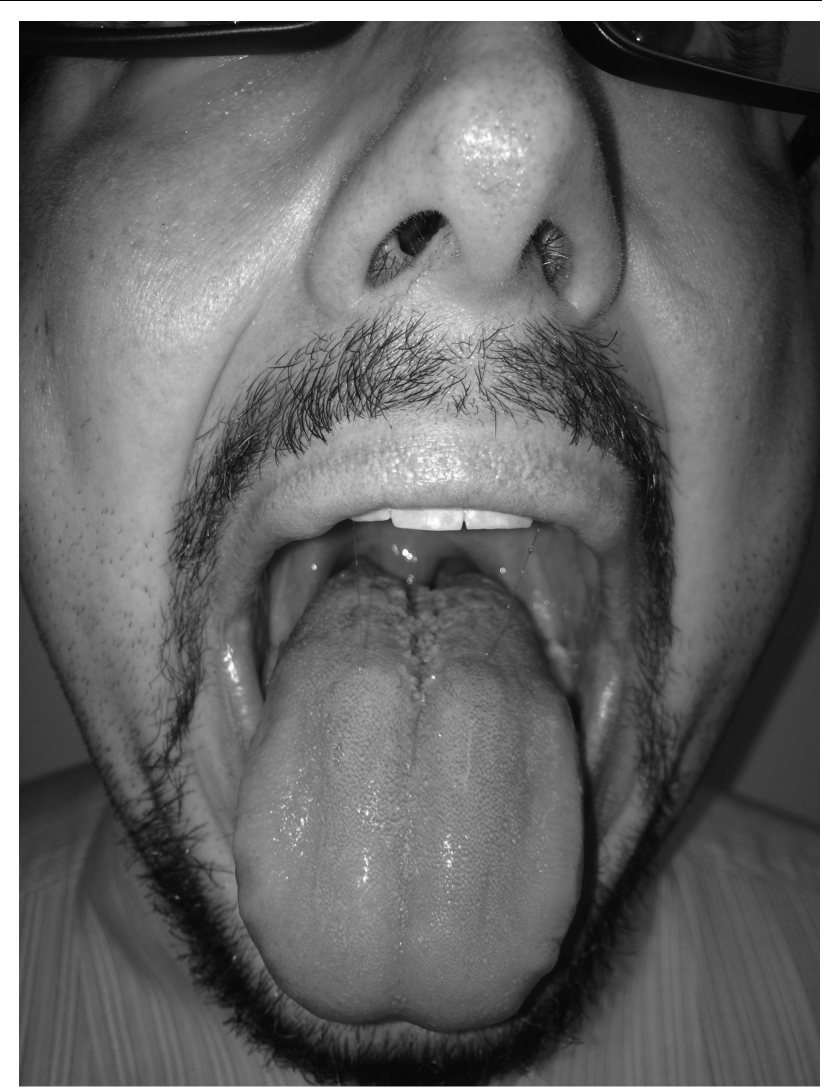

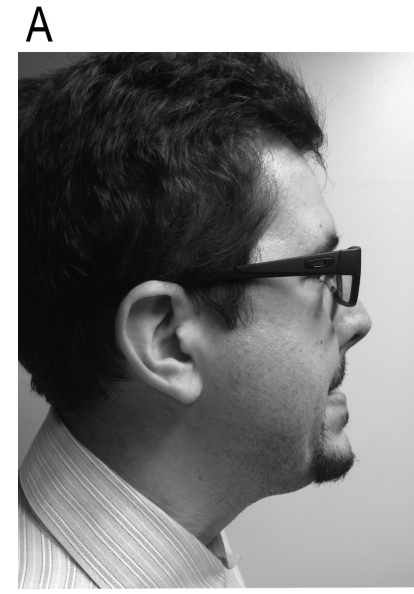

B

Fig. 1. The patient sits in an upright position to obtain all photographs. A - the patient opens his mouth as wide as possible while sitting in the upright position; $\mathrm{B}$ - the patient bites his upper lip, demonstrating the ability to prognath; $\mathrm{C}$ - an example of the patient maximally extending his neck without pain

was waived by the IRB for the survey, as consent was implied by participation. After reading the email invitation, clinical anesthesia providers who had seen the airway photographs from the UF Department of Anesthesiology were surveyed using Survey Monkey. Any anesthesia providers who had not seen the airway photographs were surveyed using a truncated version of the same survey. Reminder emails were sent out at 
2 and 4 weeks after the initial email invitation. The survey was closed 6 weeks after the original email invitation. A chi-square test for goodness of fit was used to assess if the observed frequencies in responses to each question significantly differed from the distribution that would be predicted by chance (evenly distributed across categories). Chi-square tests for independence were used to evaluate if the responses to each question differed due to the type of provider or years in practice. $P<0.05$ was considered statistically significant. Formal feedback was not sought from the MTs.

\section{Results}

There were $145(80.6 \%)$ respondents of the 180 individuals who were emailed. Forty-nine attendings $(33.8 \%)$ and 64 residents $(44.1 \%)$ accounted for most of the responses, with over one-half $(\mathrm{n}=99$ or $68.3 \%)$ reporting 5 or fewer years in practice (Table 1). Of the 145 respondents, $108(74.5 \%)$ said they had viewed the photographs in the preanesthetic note and these results are included in the final analysis, while 37 $(25.5 \%)$ reported that they had not viewed the airway photographs (Table 1).

Table 1. Respondent demographics

\begin{tabular}{lc}
\hline & $\%$ \\
\hline $\begin{array}{l}\text { Are you currently a: } \\
\quad \text { Resident }\end{array}$ & 44.1 \\
$\quad$ Anesthesia Assistant & 10.3 \\
\hline$\quad$ Nurse Anesthetist & 11.7 \\
$\quad$ Attending & 33.8 \\
\hline $\begin{array}{l}\text { How many years have you been in practice? } \\
\quad \text { 0-5 years }\end{array}$ & 69.3 \\
\hline 6-10 years & 13.8 \\
\hline 11+ years & 17.9 \\
\hline $\begin{array}{l}\text { Have you seen the pictures of the airway assessment on } \\
\text { patients you have taken care of in the operating room? (if } \\
\text { no, then please skip the rest of the questions and submit }\end{array}$ \\
$\begin{array}{l}\text { your survey) } \\
\quad \text { Yes }\end{array}$ \\
$\quad$ No & 74.5 \\
\hline
\end{tabular}

The majority of those surveyed responded that the photographs aided the development of a preliminary anesthesia plan before patient arrival $\left(\chi^{2}=55.7, \mathrm{df}=\right.$ $4, P<0.0001$ ) (Fig. 2) and that the photographs improved satisfaction with the quality of the preoperative airway assessment $\left(\chi^{2}=53.0, \mathrm{df}=4, P<0.0001\right)$ (Fig. 3). Furthermore, over $80 \%$ of respondents reported that they preferred preoperative evaluation with photographs of the airway assessment $\left(\chi^{2}=114.7\right.$, $\mathrm{df}=2, P<0.0001)$ (Fig. 4). There were no significant differences $(P>0.05)$ in the survey responses related to type of provider or years of practice.

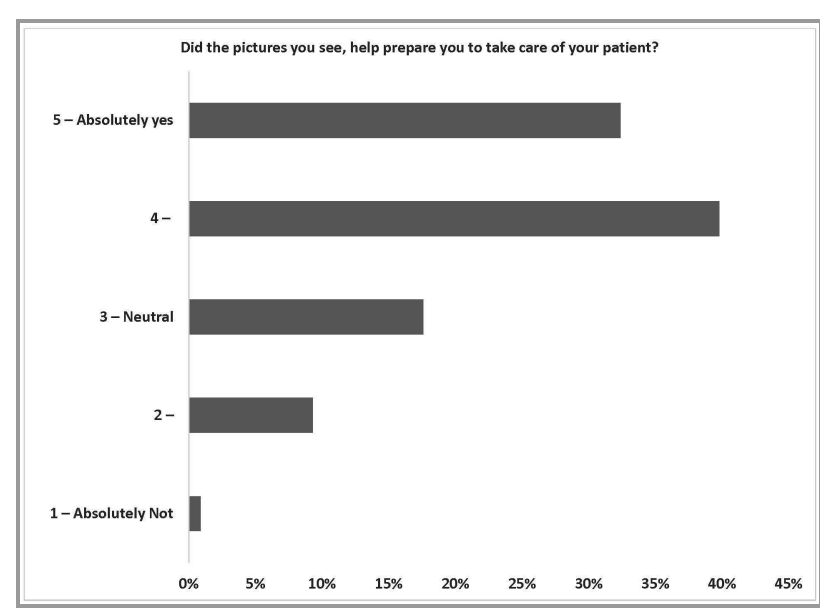

Fig. 2. Results from question, "Did the pictures you see help prepare you to take care of your patient?"

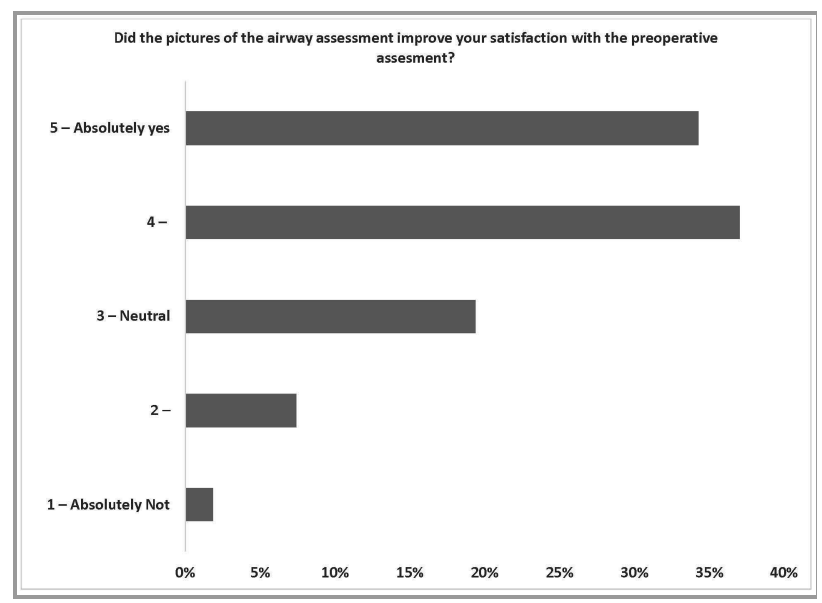

Fig. 3. Results from question, "Did the pictures of the airway assessment improve your satisfaction with the preoperative assessment?"

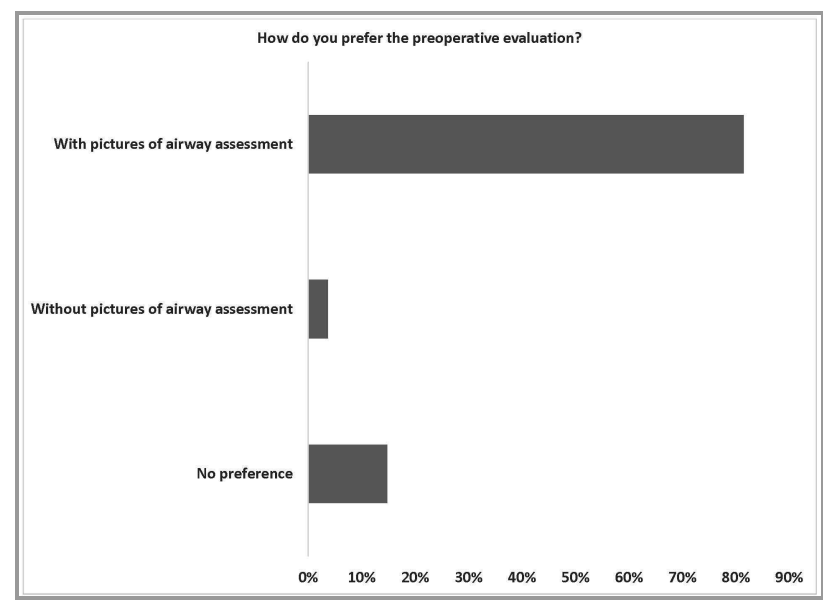

Fig. 4. Results from question, "How do you prefer the preoperative evaluation?" 


\section{Discussion}

The airway assessment photographs in the presurgical evaluation were rated as useful and as improving satisfaction with the preoperative anesthesia evaluation by a significant majority of the responding anesthesia providers in this study. In modern practice, it is increasingly common that the providers who actually administer the anesthetic are different from the providers performing the initial preanesthesia assessment. The incorporation of airway photography could be helpful for the actual anesthetist providing anesthesia for the patient if a different person made the preoperative assessment. We conclude that the airway photographs are perceived to provide a less ambiguous and thereby more informative assessment of the potential difficulty or ease of the patient's airway for the assigned anesthesia provider before surgery and provides another layer of information to incorporate into the preoperative planning for a patient's anesthetic. Presenting the traditional descriptive airway assessment with the addition of photographs provides nuanced information about the patient's habitus and an examination that is often difficult or impossible to completely relay to another provider in the traditional preanesthesia assessment. The photographs allow each practitioner to be his or her own observer rather than relying on another individual's airway assessment. The airway photographs are of course not intended to, and do not, replace the immediate preprocedure physical assessment done by the actual bedside provider. Over time, enough photographs may accumulate to analyze how airway examinations may change as a result of weight gain or loss and with the progression of disease processes.

In addition to survey data, we received unsolicited feedback from several faculties. One faculty member contacted a patient the day before the procedure to provide instructions for him to shave his beard. The attending anesthetist stated the photographs were very helpful and had raised concerns regarding the ability to mask ventilate the patient; removing the beard would facilitate a better mask seal during ventilation [4-6]. Another commented that the pictures were much more valuable for preassessment of a potential difficult airway than the previous written descriptions and Mallampati assessments. A third remarked that the pictures helped him to prepare for cases in the magnetic resonance imaging suite that were often performed for tumor evaluation and thus elevated concern for a potentially difficult airway in a non-operating room anesthetizing location. The photographs stimulated a plan to have a difficult airway cart for this case in this remote location.

There were several barriers encountered and addressed during the implementation of this assessment enhancement. The first was that photographs, which fall under protected health information, may have previously been taken on personal devices without patient knowledge or approval [7-10]. Our policy on photography during patient care was reviewed to ensure that the presurgical clinic was in compliance with local and HIPAA standards. The second barrier to implementation was acquiring the appropriate permissions from the Information Technology department, which involved several different processes. These included obtaining medical record access for the MTs, installation of the EMR software (EPIC) on departmentally owned iPads, and the ability for multiple users to access Epic's software on the same mobile device. A third barrier was finding a light source good enough to ensure high-quality photographs. After trying a variety of light sources, a Verso Clip Light for EReaders (LightWedge, LLC, Newton, MA) was used. This clip light attaches to the iPad, freeing the MTs' hands to focus on obtaining the photographs.A fourth barrier to implementation was some initial resistance to the additional workload on the MTs within the workflow. Finally, there was and is a learning curve with taking high-quality airway assessment photographs. The MTs needed instruction focused on the important aspects that were required to obtain a high-value airway photograph (e.g., ensuring uvula visualization for the Mallampati or the upper lip visible for prognath). The staff were also instructed to get "uncomfortably close". After several months, the MTs required a review of the elements that constitute high-quality airway assessment photographs.

Another potential barrier is patient refusal or inability to have their photograph taken in the three different poses. While extremely rare, several patients have refused to have their pictures taken. The inability to comply with the three airway exam maneuvers due to patient age or developmental delay was much more frequent, but still relatively infrequent. These patients, similarly, were unable to comply with a bedside exam. Our clinic did not keep track of the number of patients who either refused or were unable to have their photographs taken in the three poses.

A theoretical barrier to implementating photography of the airway assessment is the increased time it takes to $\log$ into EPIC and obtain the three images. This extra time ended up not being significant as we were able to accomplish this task during the time the vital signs were being taken. Thus, an increase in the "door to door" clinic time was not experienced by patients.

There are several limitations to this study. First, this is a survey of the opinions of one anesthesiology department. It is possible that other providers of anesthesia may not find the images as helpful. Another limitation of the study is, based on the responses in the 
free text portion of the survey. It was obvious that some clinical anesthesia providers had clicked the link saying that they had not seen the airway photographs, but had in reality seen the photographs. The analysis of the results was made on an "intention to treat" basis, and only responses for providers who clicked the link for seeing the photographs were included in the analysis of this study. A possible limitation of this study is the response rate or "cooperation" rate of $80.6 \%$ possibly leading to sampling bias. Cummings, Savitz, and Konrad reported an average response rate of $61 \%$ for mailed physician surveys [11], and Nulty describes multiple studies where online survey response rates are usually lower than paper surveys [12]. Our reported response rate is actually $80.6 \%$ of a studied population, and not a portion of a random sampling from a population, thus minimizing the possibility of sampling bias.

Two other areas of improvement would be: 1) having a ruler next to the mouth opening and a protractor next to the neck extension photographs, which would provide even more objective data and 2) a video of the physical exam, which would provide the observer with more dynamic information on the patients' mobility. However, this feature is not supported by the EPIC Canto software.

\section{Conclusions}

We described the successful implementation of preoperative airway examination photographs into the preanesthesia assessment in a busy presurgical clinic. Clincal anesthesia providers overwhelmingly found value in this preassessment airway enhancement through photographs and anecdotally changed their preparations based on some of the photographs. Barriers to implementation included permissions from information technology, training of personnel, and optimizing the photography equipment. After implementation, there was improvement in the clinic anesthesia provider's satisfaction with the preanesthesia assessment and a demand for use on a wider scale. The implementation should be possible for any institution using EPIC for their EMR and may be able to be used with other EMRs as well.

\section{Conflict of interest}

Nothing to declare

\section{Funding}

This work was supported by internal funding from the Department of Anesthesiology, College of Medicine, University of Florida

\section{References}

1. Mallampati SR, Gatt SP, Gugino LD, Desai SP, Waraksa B, Freiberger D, et al. A clinical sign to predict difficult tracheal intubation: a prospective study. Can Anaesth Soc J 1985; 32: 429-434

2. Samsoon GL, Young JR. Difficult tracheal intubation: a retrospective study. Anaesthesia 1987; 42: 487-490. DOI: 10.1111/j.1365-2044.1987.tb04039.x

3. Connor CW, Segal S. The importance of subjective facial appearance on the ability of anesthesiologists to predict difficult intubation. Anesth Analg 2014; 118: 419-427. DOI: 10.1213/ ANE.0000000000000012

4. Kheterpal S, Han R, Tremper KK, Shanks A, Tait AR, O’Reilly $\mathrm{M}$, et al. Incidence and predictors of difficult and impossible mask ventilation. Anesthesiology 2006; 105: 885-891

5. Johnson JO, Bradway JA, Blood T. A hairy situation. Anesthesiology 1999; 91: 595

6. Butterworth IV JF, Mackey DC, Wasnick JD. Airway management. In: Butterworth IV JF, Mackey DC, Wasnick JD, eds. Morgan \& Mikhail's Clinical Anesthesiology. $5^{\text {th }}$ edition. New York: McGraw-Hill; 2013: 309-341

7. Cohen R. When med students post patient pictures. The New York Times Magazine [online]. 2011 [cited 2015, December 19]. Available from: http://www.nytimes.com/2011/02/13/ magazine/13FOB-Ethicist-t.html?_r $=0$

8. Mann D. Medical students reckless on Internet, sometimes at patients' expense. CNN [online]. 2009 [cited 2015, December 19]. Available from: http://www.cnn.com/2009/HEALTH/09/ $22 /$ medical.students.internet/index.html?eref=rss_latest

9. Bianco R. Elder care workers mock patient, post video on Snapchat. ABC 10 News [online]. 2015 [cited 2015, December 19]. Available from: http://www.10news.com/news/nursinghome-nurses-mock-patient-post-video-on-snapchat

10. FierceHealthcare. Photos of dying patient posted to Facebook get four hospital workers fired. 2010 [cited 2015, December 19]. Available from: http://www.fiercehealthcare.com/story/ facebook-firings-photos-dying-patient-get-four-hospitalworkers-fired-three-disciplined/2010-0

11. Cummings SM, Savitz LA, Konrad TR. Reported response rates to mailed physician questionnaires. Health Serv Res 2001; 35: 1347-1355

12. Nulty DD. The adequacy of response rates to online and paper surveys: what can be done? Assess Eval High Educ 2008; 33: 301-314. DOI: 10.1080/02602930701293231 